\title{
ORIGINAL
}

\section{FACTORES ASOCIADOS A PRESENTAR PARADA RESPIRATORIA EN LAS SOBREDOSIS POR OPIÁCEOS ATENDIDAS EN UN ESCENARIO ABIERTO DE CONSUMO DE DROGAS EN BARCELONA}

Manel Anoro (1), Enrique Ilundain (1), Rosa Rodriguez (1), Lali Rossell (1), Begoña Iglesias (2), Caterina Guinovart (2), Mercedes Gabari (2)

(1) Programa socio sanitario Can Tunis. Associació Benestar i Desenvolupament.

(2) Departamento de Epidemiología y Salud Pública. SAP Ciutat Vella, Barcelona.

\section{RESUMEN}

Fundamento: Determinar los factores que se asocian a parada respiratoria en las sobredosis por opiáceos (coma, miosis pupilar, depresión respiratoria y respuesta a naloxona) atendidas en el barrio de Can Tunis de Barcelona.

Métodos: Se realizó un estudio observacional transversal, donde se incluyeron todas las sobredosis por opiáceos atendidas entre marzo del 2001 y junio del 2002. Se registraron las características previas del paciente, las del consumo de opiáceos que produjo la sobredosis, el consumo concomitante de otras substancias y la clínica, mediante una hoja de recogida de datos cumplimentada después de atender la sobredosis. En el análisis se realizó una regresión logística.

Resultados: De las 222 sobredosis atendidas un 60'8\% presentaron parada respiratoria. Se asoció a ella no haber consumido heroína
en los últimas 2 semanas (OR: 1,$893 ; \mathrm{p}=0,04)$ y no haber consumido en los últimas 2 semanas (OR: 1,$893 ; \mathrm{p}=0,04)$ y no haber consumido
previamente benzodiazepinas (OR: 0,$462 ; \mathrm{p}=0,017)$. El consumo de alcohol, cocaína o metadona no predispondría a la aparición de parada respiratoria.

Conclusiones: Se identifica como factor de riesgo principal para sufrir una parada respiratoria en una sobredosis por opiáceos una abstinencia previa superior a quince días. Por otra parte, el consumo de benzodiazepinas se asocia con la ausencia de parada respiratoria en la sobredosis. Sería interesante analizar otros factores, que podríen la sobredosis. Sería interesante analizar otros factores, que podrí-
an confundir nuestros resultados y que no han sido incluidos en el estudio, asociados tanto al consumo de benzodiazepinas como al hecho de no presentar parada respiratoria en una sobredosis.

Palabras clave: Drogadicción. Sobredosis. Heroína. Factores de riesgo. Prevención. Naloxona.

Correspondencia:

Manel Anoro Preminger

ABS Raval Sud

Avda Drassanes 17-21 (7 $7^{\mathrm{a}}$ planta)

08001 Barcelona

Correo electrónico: 31885map@ comb.es

\section{ABSTRACT}

Factors Related to Experiencing Respiratory Failure in Cases of Opiate Overdose for which Care Was Provided in an Open Setting. Barcelona, Spain

Background: To determine the factors associated with respiratory arrest in opiate overdoses (coma, pupillary miosis, respiratory depression, and response to naloxone) among injecting drug users in the Can Tunis quarter of Barcelona.

Methods: We ran a transversal observational study where all overdoses assisted between March, 2001 and June, 2002. After overdose treatment, data were collected using a standard questionnaire, including: patients' sociodemographic data, opiate and other substances' use prior to overdose, clinical signs and symptoms presented, and medical intervention received, by ways of a standardised questionnaire. Logistic regression was used as a tool for analysis.

Results: Of 222 opiate overdose cases, $60.8 \%$ showed respiratory arrest. Of all risk factors tested, only prior abstinence heroin abstinence for 2 weeks or longer days $(\mathrm{OR}=1.893 ; \mathrm{p}=0.04)$, and no previous consumption of benzodiazepines (OR:0,462; $\mathrm{p}=0,017$ ), proved to have a statistically significant association with suffering a respiratory arrest. Concomitant use of alcohol, cocaine or methadone appeared not associated with suffering respiratory arrest in opiate overdose.

Conclusions: The main risk factor for respiratory arrest in opiate overdoses was a prior abstinence period of more than 2 weeks. Benzodiazepines use was associated with absence of respiratory arrest in overdose cases. Alcohol or methadone use, as well as the use of larger quantities of heroin, was not associated with suffering respiratory arrest in opiate overdoses. A study of other factors, not included in this study, and that could interfere with our results, should be considered for their possible relationship to benzodiazepine use as well as to absence of respiratory arrest in overdose cases.

Key words: Drug Addiction. Overdose. Heroin. Apnea. Risk factors. Prevention. Naloxone. 


\section{INTRODUCCIÓN}

La mortalidad entre el colectivo de usuarios de heroína es de 20 a 30 veces mayor que la de la población general de la misma edad $^{1}$. La principal causa de muerte entre estos usuarios sigue siendo las sobredosis seguida de la infección por el VIH/SIDA ${ }^{2-4}$. No existe una definición clínica clara de lo que se entiende por sobredosis, pero podríamos catalogarla como una reacción adversa aguda secundaria a la administración de opiáceos que cursa con coma más o menos profundo, miosis pupilar y depresión respiratoria que, si es intensa, puede llevar a una parada respiratoria y a la muerte en pocos minutos $^{5}$. Estudios recientes señalan que su incidencia entre consumidores de heroína españoles sería del $10 \%{ }^{6}$. Concretamente en la ciudad de Barcelona la tasa de mortalidad por esta causa durante el periodo 1990-2000 se mantuvo estable entre $10-15$ casos por 100.000 habitantes de entre 15 y 49 años ${ }^{7}$. Si a todo ello añadimos que en el año 1999 en Barcelona representó la primera causa de muerte, entre hombres y mujeres de 25 a 34 años ${ }^{8}$, podemos asegurar que se trata de un importante problema de salud pública aún no resuelto. Se han intentado determinar los factores que pueden predisponer a un consumidor de heroína a sufrir una sobredosis ${ }^{2,9-11}$. Se han destacado factores como la pérdida de tolerancia después de un largo tiempo de abstinencia $^{4}$, el consumo concomitante de otras substancias (benzodiazepinas, alcohol o metadona $)^{2,12-14}$, el consumo de una dosis de heroína mayor a la habitual o la vía de administración endovenosa de los opiáceos. Can Tunis es el mayor espacio abierto de consumo de drogas inyectadas de la ciudad de Barcelona. La accesibilidad al barrio y la posibilidad de adquirir pequeñas cantidades de droga a bajo precio (una 'papelina' de heroína de aproximadamente $90 \mathrm{mg}$ vale 5 euros) ha convertido a Can Tunis en el mayor espacio de venta y consumo de drogas inyectadas del área metropolitana. Todo ello, hace de este barrio uno de los puntos con mayor incidencia de sobredosis por opiáceos de la ciudad. En este espacio abierto se produce, aproximadamente, una sobredosis cada dos días. Nuestra experiencia en el terreno nos sugiere que resulta fundamental diferenciar aquellas sobredosis que cursan con signos graves (parada respiratoria) y, por lo tanto, potencialmente mortales, de aquellas que entrando dentro de la definición general de sobredosis, probablemente no tendrán un desenlace fatal. Creímos interesante determinar la implicación de los distintos factores de riesgo referidos en la literatu$\mathrm{ra}^{2,5,9-11,15}$ en el desenlace de las sobredosis atendidas en nuestro centro ya que los estudios realizados al respecto se han llevado a cabo en un ámbito diferente al nuestro ${ }^{6,16}$.

El objetivo de este estudio es identificar los factores asociados a sufrir una parada respiratoria en los usuarios de drogas que presentaron sobredosis por opiáceos.

\section{SUJETOS Y MÉTODOS}

Se realizó un estudio descriptivo observacional transversal. Nuestro equipo de salud (un médico y tres diplomados en enfermería) trabaja en el barrio de Can Tunis de Barcelona durante 6 horas al día en horario de mañana todos los días del año. Debido a nuestra proximidad a las áreas de consumo y a la accesibilidad del recurso, recibimos varios avisos de emergencias cada día. No todas ellas son verdaderas emergencias, pero siempre que se recibe un aviso dos miembros del equipo (un médico y una enfermera o dos enfermeras) acuden al lugar realizando una evaluación de la situación clínica e iniciando tratamiento si es necesario. Dado que muchos de estos casos de sobredosis son atendidos por nuestro equipo sanitario, se decidió incluir en el estudio todas las sobredosis por opiáceos atendidas durante el periodo comprendido entre marzo del 2001 y junio del 2002 (222 sobredosis). Únicamente se excluyeron del estudio los eventos que no cumplían los criterios clínicos de sobredosis por opiáceos. La parada respira- 
toria fue definida como la ausencia de movimientos respiratorios espontáneos por parte del paciente a nuestra llegada.

Una vez recibido el aviso de sobredosis, dos personas del equipo sanitario se desplazaban al lugar donde esta se había producido. Confirmado el diagnóstico de sobredosis por opiáceos se iniciaban las maniobras de soporte vital y la administración de naloxona para revertir el cuadro clínico. Una vez recuperado, el paciente era remitido a nuestro centro para continuar en observación. Si el paciente había cumplido criterios clínicos de sobredosis por opiáceos (coma, miosis, depresión respiratoria y respuesta a la naloxona) se incluía en el estudio y se procedía a cumplimentar la hoja de recogida de datos por parte del mismo profesional que había atendido la sobredosis.

Para todas las sobredosis incluidas en el estudio (n=222) se recogía: sexo (0:hombre, 1:mujer); edad (0:27, 1:28-30, 2:31-35; 3:36 años); consumo concomitante de cocaína (0:heroína + cocaína, 1:heroína sola); cantidad en «papelinas» (0:1 papelina, 1: 2 o más); tiempo en días desde el último consumo de heroína (0:<15 días, 1: 15 días); consumo previo de benzodiazepinas, alcohol o metadona (0:No, 1: Sí); minutos transcurridos entre el aviso y la atención a la sobredosis $(0: 5,1:>5)$ y la gravedad del cuadro (0: respiración espontánea, 1: parada respiratoria).

En el análisis bivariado, para determinar diferencias estadísticamente significativas de variables cualitativas, se ha utilizado la prueba de $\chi^{2}$ comprobando las condiciones de aplicación (casos esperados <5), de no cumplirse se utilizaba la prueba exacta de Fisher. Para determinar diferencias en variables cuantitativas con datos independientes se ha utilizado la prueba de T de Student, comprobando previamente que se cumplen los criterios de aplicación (igualdad de varianzas con la F de Snedecor y normalidad).
Para determinar la fuerza de la asociación se estimó la odds ratio (OR) con intervalos de confianza del 95\%. A nivel multivariante se realizó una regresión logística, en la que la variable dependiente era la presencia o ausencia de paro respiratorio como variable que definía la gravedad de la sobredosis. Se incluyeron como variables independientes todas las que a nivel bivariante presentaban como mínimo significación marginal $(\mathrm{p}<0,15)$. Se ha utilizado el procedimiento stepwise, el criterio de entrada de la variable ha sido $\mathrm{p}<0,05$ y el de salida $\mathrm{p}>0,10$. La bondad del modelo se determinó con el estadístico -2log likehood y el porcentaje global de buena clasificación. La significación de cada una de las variables se determinó utilizando el estadístico Wald.

\section{RESULTADOS}

De los 222 casos de sobredosis atendidos, $135(60,8 \%)$ fueron catalogados como graves (con parada respiratoria). La vía de administración de la heroína fue la endovenosa en todas las sobredosis incluidas y en ninguna de ellas se produjo la muerte del paciente. En los casos de sobredosis analizados, el sexo $(\mathrm{p}=0,671)$, la edad del paciente $(\mathrm{p}=0,505)$, el consumo previo de alcohol $(\mathrm{p}=0,549)$, el consumo concomitante de cocaína ( $\mathrm{p}=0,108)$, la cantidad de heroína inyectada $(\mathrm{p}=0,565)$ o el tiempo transcurrido entre el aviso al equipo sanitario y el inicio de la atención al paciente $(\mathrm{p}=0,278)$ no se relacionaron con presentar un cuadro con parada respiratoria. Sin embargo, llevar más de 15 días sin consumir (OR: 2,18; $\mathrm{p}=0,011$ ) estaba asociado a sufrir un evento más grave (con parada respiratoria). La media de días de abstinencia era de 21,2 días (DE:34,4) en los pacientes que no presentaron parada respiratoria y de 38,6 días (DE:43,8) en los que si la sufrieron $(\mathrm{p}=0,002)$. Por el contrario, el consumo de benzodiazepinas el mismo día de la sobredosis se asociaba a que ésta no presentara parada respiratoria $(\mathrm{p}=0,002)$ (tabla 1). 
Tabla 1

Análisis bivariante de las variables incluidas en el estudio y su relación con la presencia o no de parada respiratoria en la sobredosis

\begin{tabular}{|c|c|c|c|c|c|}
\hline \multirow{2}{*}{ Variable } & \multicolumn{2}{|c|}{ Respiración espontanea (n=87) } & \multicolumn{2}{|c|}{ Parada respiratoria $(\mathrm{n}=135)$} & \multirow{2}{*}{$\begin{array}{c}\text { OR (IC 95\%) } \\
\text { Significación estadística }\end{array}$} \\
\hline & número & $\%$ & número & $\%$ & \\
\hline \multicolumn{6}{|l|}{ Sexo } \\
\hline Hombre & 75 & 38,7 & 119 & 61,3 & \multirow{2}{*}{$\begin{array}{c}0,84(0,38-1,87) \\
\mathrm{p}=0,67\end{array}$} \\
\hline Mujer & 12 & 42,9 & 16 & 57,8 & \\
\hline \multicolumn{6}{|l|}{ Consumo Metadona $^{*}$} \\
\hline No & 83 & 38,4 & 133 & 61,6 & \multirow{2}{*}{$\begin{array}{c}0,16(0,02-1,42) \\
\mathrm{p}=0,08\end{array}$} \\
\hline $\mathrm{Si}$ & 4 & 80,0 & 1 & 20,0 & \\
\hline \multicolumn{6}{|c|}{ Consumo previo benzodiazepinas $\dagger$} \\
\hline No & 46 & 31,9 & 98 & 68,1 & \multirow{2}{*}{$\begin{array}{c}0,40(0,23-0,71) \\
\mathrm{p}<0,01\end{array}$} \\
\hline Sí & 41 & 53,9 & 35 & 46,1 & \\
\hline \multicolumn{6}{|l|}{ Consumo previo alcohol } \\
\hline No & 65 & 38,5 & 104 & 61,5 & \multirow{2}{*}{$\begin{array}{c}0,82(0,44-1,56) \\
\mathrm{p}=0,55\end{array}$} \\
\hline Sí & 22 & 43,1 & 29 & 56,9 & \\
\hline \multicolumn{6}{|l|}{ Heroína más cocaína } \\
\hline Heroína + cocaína & 42 & 34,4 & 80 & 65,6 & \multirow{2}{*}{$\begin{array}{c}0,64(0,37-1,10) \\
p=0,11\end{array}$} \\
\hline Heroína sola & 45 & 45,0 & 55 & 55,0 & \\
\hline \multicolumn{6}{|l|}{ Días previos abstinencia $\uparrow$} \\
\hline$<15$ días & 47 & 45,2 & 57 & 54,8 & \multirow{2}{*}{$\begin{array}{c}2,18(1,19-3,97) \\
\mathrm{p}=0,01\end{array}$} \\
\hline$\geq 15$ días & 25 & 27,5 & 66 & 72,5 & \\
\hline \multicolumn{6}{|l|}{ Edad } \\
\hline$\leq 27$ años & 27 & 42,9 & 36 & 57,1 & \\
\hline $28-30$ años & 17 & 47,2 & 19 & 52,8 & $0,84(0,37-1,91)$ \\
\hline $31-35$ años & 27 & 36,0 & 48 & 64,0 & $1,33(0,67-2,65)$ \\
\hline$\geq 36$ años & 16 & 33,3 & 32 & 66,7 & $\begin{array}{c}1,50(0,69-3,27) \\
p=0,51\end{array}$ \\
\hline \multicolumn{6}{|c|}{ Minutos de demora en la asistencia } \\
\hline$\leq 5$ minutos & 79 & 40,5 & 116 & 59,5 & \multirow{2}{*}{$\begin{array}{c}1,62(0,67-3,88) \\
\mathrm{p}=0,28\end{array}$} \\
\hline$>5$ minutos & 8 & 29,6 & 19 & 70,4 & \\
\hline \multicolumn{6}{|c|}{ Cantidad de heroína en "papelinas" } \\
\hline 1 "papelina" & 50 & 37,3 & 84 & 62,7 & \multirow{2}{*}{$\begin{array}{c}0,83(0,45-1,55) \\
\mathrm{p}=0,57\end{array}$} \\
\hline$\geq 2$ "papelinas" & 25 & 41,7 & 35 & 58,3 & \\
\hline
\end{tabular}

OR: odds ratio. IC: intervalo de confianza. * Significación marginal $(\mathrm{p} \leq 0,15)$. $†$ Significación estadística $(\mathrm{p} \leq 0,05)$ Los porcentajes han sido calculados por filas. DE: Desviación estandar

En el análisis multivariante, y tras introducir en el modelo el consumo de metadona, consumo previo de benzodiazepinas y días previos de abstinencia, el hecho de presentar parada respiratoria en la sobredosis se asoció a no haber consumido heroína en los últimas 2 semanas (OR: 1,$893 ; \mathrm{p}=0,04)$ y a no haber consumido previamente benzodiazepinas (OR: 0,462; p=0,017). El modelo clasifica adecuadamente las sobredosis con parada respiratoria ( $83,5 \%$ de buena clasificación) pero presenta deficiencias importantes en la clasificación de las que no cursan con parada respiratoria (33,3\% de buena clasificación) (tabla 2).

\section{DISCUSIÓN}

La división entre sobredosis mortales y no mortales se utiliza con frecuencia en la literatura ${ }^{10,17,18}$ y es adecuada para estudiar qué factores llevarían a uno u otro desenlace. Sin embargo, los trabajos realizados hasta ahora 
Tabla 2

Modelo final del análisis multivariante de las variables asociadas a la presencia o no de parada respiratoria en las sobredosis

\begin{tabular}{|l|c|c|c|c|c|}
\hline \multicolumn{1}{|c|}{ Variables } & B & Wald & p & Exp (B) & 95\% CI Exp (B) \\
\hline Constante & 0,510 & 4,283 & 0,038 & 1,665 & \\
\hline Consumo previo benzodiazepinas & $-0,771$ & 5,697 & 0,017 & 0,462 & $0,245-0,871$ \\
\hline Días previos abstinencia & 0,638 & 4,066 & 0,04 & 1,893 & $1,018-3,520$ \\
\hline
\end{tabular}

-2log likelihood: 242,65. Porcentaje de correcta clasificación: 64,8\% (33,3\% para respiración espontánea y 83,5\% para parada respiratoria). Se habían introducido en el modelo el consumo de metadona, consumo previo de benzodiazepinas y días previos de abstinencia.

se han centrado en estudiar únicamente uno de los dos grupos. Así, las sobredosis mortales se recogían en estudios forenses ${ }^{19,20}$ y las no fatales mediante estudios retrospectivos de usuarios con consumo activo o en tratamiento ${ }^{6}$. Desde esta perspectiva resulta difícil, basándose en la literatura, determinar los factores asociados al desenlace final de una sobredosis. Nuestro ámbito de trabajo (gran número de sobredosis, diagnóstico y tratamiento por parte de personal médico y observación posterior del paciente) nos ha permitido acceder a la mayor parte de las sobredosis producidas en la zona y a realizar un adecuado tratamiento de las mismas.

El trabajo presenta indudables limitaciones. Recogida de datos mediante encuesta después del evento, no disponer de instrumentos de medición más fiables para determinar substancias consumidas previamente o no saber, de no ser por nuestra actuación, qué casos hubieran tenido como desenlace la muerte del individuo. Además, el tiempo desde el último consumo de heroína puede verse afectado por un sesgo de recuerdo, sobre todo cuando el último consumo fue muy anterior. Estos pacientes acostumbran a referir este periodo en meses y no en días y así la precisión de la variable puede ser menor. Esto implica que el riesgo de padecer una parada respiratoria en cada uno de los grupos no se pueda cuantificar con precisión. También cabe señalar que nuestro estudio está realizado en el terreno y esto dificul- ta la comparación con los resultados de otros estudios que mayoritariamente se han hecho en otros ámbitos (principalmente hospitalario y forense $)^{19}$. Finalmente hay que resaltar la dificultad de comparar nuestros resultados con los de otro tipo de estudios que no se realizan en el momento en que se produce la sobredosis $^{6,20}$. A pesar de todo ello, creemos que el estudio nos ha permitido analizar adecuadamente los determinantes de la gravedad de la sobredosis.

La pérdida de tolerancia a la heroína debida a la abstinencia en su consumo ha sido señalado en la literatura como uno de los principales factores de riesgo para sufrir una sobredosis ${ }^{2,10}$. El estudio llevado a cabo identifica como factor de riesgo para sufrir una parada respiratoria en una sobredosis por opiáceos y probablemente mortal, una abstinencia previa superior a quince días. Los casos que presentaron parada respiratoria serían, con mucha probabilidad, sujetos con un importante consumo anterior (alta tolerancia previa), abstinentes durante un tiempo (más de quince días), sin tratamiento con agonistas opiáceos ni otra medicación y que se mostrarían hipersensibles a la heroína.

Destaca también como resultado, en contra de lo que se concluye en otros trabajos $^{12,14,20,23}$, la nula influencia de otras sustancias consumidas en la aparición de una parada respiratoria en la sobredosis. El con- 
sumo de depresores como benzodiazepinas, alcohol o metadona, o de estimulantes como la cocaína, no predispondrían a presentar un cuadro de mayor gravedad. Además, en nuestro estudio el consumo de benzodiazepinas se asocia con la ausencia de parada respiratoria en la sobredosis. Podría suceder que el consumo de benzodiazepinas se asocie positivamente a presentar una sobredosis, tanto sin parada respiratoria como con ella, y que esta asociación fuera más fuerte en las primeras.

Por otra parte, existen factores asociados al consumo de benzodiazepinas y a presentar una sobredosis no grave, que no han sido incluidos en el estudio. En los estudios diseñados para determinar factores asociados a un evento, se suele hacer una recogida exhaustiva de los factores de riesgo, pero existe la tendencia a no incluir los factores protectores. Probablemente nuestro estudio presenta también un sesgo de este tipo. De hecho el modelo clasifica bien las sobredosis graves pero no lo hace en el caso de las no graves.

La relación entre consumo de benzodiazepinas y sobredosis no grave abre interesantes incógnitas:

1. ¿Es la definición de sobredosis aceptada hasta ahora demasiado amplia? Probablemente en el grupo de sobredosis no graves se mezcló un abanico de cuadros clínicos, todos ellos dentro de la definición de sobredosis, que respondían a múltiples causas; entre ellas la mezcla de heroína con otros fármacos en consumidores habituales de múltiples sustancias y con una elevada tolerancia a todas ellas. Se trataría de sobredosis por opiáceos no puras, menos graves y representarían el grueso de los estudios retrospectivos realizados hasta ahora ${ }^{6}$.

2. ¿Hasta qué punto los depresores del sistema nervioso central no opiáceos consumidos pueden favorecer una parada respiratoria?

Desde el inicio de nuestra experiencia en la atención a sobredosis intuimos que los eventos más graves eran los que se producían con mayor rapidez y los que mejor respondían a la naloxona. En este sentido las sobredosis graves observadas en nuestro lugar de venopución higiénica (EVA Espacio de Venopunción higiénica Asistida) nos han aportado valiosa información y muestran un paciente que entra en parada respiratoria a los pocos segundos del consumo y que revierte totalmente el cuadro clínico con el uso de naloxona. Probablemente la heroína es, con mucha diferencia, la principal causante, si no la única, de la parada respiratoria que conducirá a la muerte. Las dosis necesarias de alcohol o benzodiazepinas para producir una depresión respiratoria que conduzca a la muerte son muy elevadas y difícilmente las habrá consumido el paciente. Parece que en nuestro ámbito su consumo en dosis habituales no es decisivo en la aparición de una parada respiratoria.

Estudios realizados desde Servicios de Urgencias Hospitalarias ${ }^{22,23}$ detectan un elevado número de pacientes atendidos por sobredosis, con consumo concomitante de benzodiazepinas. Es posible que a los servicios de urgencias hospitalarias lleguen un mayor número de sobredosis no graves y se pierdan parte de las que nosotros catalogamos como graves. Es decir, algunos de los casos más graves no se derivarían al hospital ya que habrían fallecido en el lugar de la sobredosis, remitiéndose directamente para estudio forense, o bien se tratarían en el mismo lugar del evento con una buena respuesta a la naloxona, dándose el alta en la misma calle (práctica, por otra parte, muy frecuente entre los servicios extrahospitalarios de emergencias).

Por otro lado, los estudios foren$\operatorname{ses}^{13,14,20,21,24}$ también relatan un elevado número de casos con presencia en sangre $\mathrm{y}$ 
orina de metabolitos de heroína y de otras sustancias depresoras. Se trataría de estudios con resultados difícilmente extrapolables al terreno. Los criterios de selección de casos, el desconocimiento de los antecedentes personales y las circunstancias previas a la muerte del sujeto, o la tendencia a asumir la sobredosis por opiáceos como causa del óbito por el hecho de haber encontrado metabolitos de heroína en sangre, podrían esconder sesgos que restarían validez externa a los resultados.

El papel que desempeñan las benzodiazepinas en las sobredosis por opiáceos es, seguramente, complejo y no ha sido suficientemente estudiado, pero los resultados obtenidos y nuestra experiencia en el terreno nos orientan a plantear que en nuestro ámbito de trabajo la pérdida de tolerancia física a la heroína secundaria a la abstinencia es el factor determinante para sufrir una sobredosis grave, y que otros factores, como el consumo previo de otras sustancias o aumento de la dosis, no resultan decisivos. Así, el perfil de usuario con alta probabilidad de sufrir una sobredosis fatal sería aquel que llevando un tiempo sin consumir decide reiniciar el consumo de heroína. Las causas de este nuevo consumo seguramente son complejas y varían de un individuo a otro, pero estados de ánimo más depresivos, periodos de estrés, dificultades en la solución de problemas personales y, en algunos casos, cierta ideación suicida tendrían mayor relevancia que los deseos de pasarlo bien.

Se hace patente la necesidad de definir estrategias dirigidas a prevenir nuevos casos de sobredosis potencialmente fatales. Intervenciones dirigidas a sujetos largamente abstinentes o con consumos muy esporádicos podrían resultar de utilidad. Desde los Centros de Atención a Drogodependencias, Centros Penitenciarios y desde los Centros de Atención Primaria, se debería realizar un seguimiento especial de aquellos individuos que hayan sido consumidores de opiáceos y, por lo tanto, en constante situación de riesgo.
El diagnóstico y tratamiento de trastornos del estado de ánimo realizados a estos niveles podrían evitar nuevos casos.

Finalmente se abren las puertas a nuevas e interesantes propuestas. Se constata la necesidad de realizar estudios posteriores que permitan determinar con mayor fiabilidad las variables que predispondrían a sufrir una sobredosis potencialmente fatal. Pero además la gravedad del cuadro clínico, su efectiva respuesta a la naloxona, la enorme seguridad de este fármaco y, sobretodo, la necesidad de atender al paciente de forma inmediata, sugiere de nuevo la posibilidad de que los consumidores de heroína dispongan de naloxona para poder utilizar sobre terceros ${ }^{25,26}$. Equipos capacitados para atender nuevos casos de manera inmediata y que distribuyan ampollas de naloxona o, mejor aún, jeringuillas precargadas con el fármaco e impartan talleres educativos a usuarios de heroína para capacitarlos sobre estrategias preventivas y de atención a sobredosis, evitarían, seguro, nuevas muertes.

\section{AGRADECIMIENTOS}

Agradecer sinceramente al resto del equipo sociosanitario de Reducción de Daños de Can Tunis de la Associació Benestar i Desenvolupament su inestimable colaboración en la atención a las sobredosis en Can Tunis. $\mathrm{Su}$ profesionalidad nos ha permitido ofrecer, en todo momento, una correcta asistencia a los pacientes que presentaron sobredosis. Sin su ayuda este estudio nunca habría sido posible.

\section{BIBLIOGRAFÍA}

1. European Monitoring Centre for Drugs and Drug Addiction (EMCDDA). 1997 Annual Report on the State of the Drugs Problem in the European Union. Lisboa: EMCDDA; 1997.

2. Darke, S \& Zador, D. Fatal heroin 'overdose'. A review. Addiction. 1996; 91: 1765-72. 
3. Orti et al. Mortality trends in a cohort of opiate addicts, Catalonia, Spain. International J Epidemiol 1996; 25:545-53.

4. Sporer KA. Acute heroin overdose. Annals of Internal Medicine 1999; 130: 584-90.

5. World Health Organization. Substance Abuse Departament. Opioid Overdose. Trends, Risk Factors, Interventions and Priorities for Action. Genève: WHO; 1998.

6. Brugal et al. Factors associated with non-fatal heroin overdose: assessing the effect of frequency and route of heroin administration. Addiction. 2002; 97: 319-27.

7. Institut Municipal de Salut Pública. Sistema d'Informació sobre Drogues. Barcelona: Institut Municipal de Salut Pública; 2000.

8. Institut Municipal de Salut Pública. Estadístiques de Salut. Mortalitat i Natalitat a la Ciutat de Barcelona 1999. Barcelona: Institut Municipal de Salut Pública; 2002.

9. Davoli M, Perucci CA, Forastiere F, et al. Risk factors for overdose mortality: a case control study within a cohort of intravenous drug users. Int J Epidemiol 1993; 22: 273-77.

10. White J M \& Irvine RJ. Mechanisms of fatal opioid overdose. Addiction 1999; 94(7): 961-72.

11. Camí J, Domingo-Salvany. Factores de riesgo en la muerte por heroína. Med Clín (Barc) 1995; 105: 405-6.

12. Ruttenber AJ, Luke JL. Heroin-related drugs: new epidemiological insights. Science 1984; 226:1420.

13. Püschel K.. Drug-related death-an update. Forensic Sci Int. 1993; 62:121-8.

14. Kaa E, Teige B. Drug-Related deaths during the 1980s. A comparative study of drug addict deaths examined at the institutes of forensic medicine in Aarhus, Denmark and Oslo, Norway. Int J Legal Med 1993;106: 5-9.

15. Levine B, Green D \& Smialek JE. The role of ethanol in heroin deaths. J. Forensic Sci 1995; 40: 808-10.
16. Jacobs I Pre-Hospital Management of Opiate Overdose in Perth, Western Australia. Western Australian Pre-Hospital Care Research Unit. Perth: WAPHCR; 2000.

17. Gossop M, Griffiths P, Powis B, Williamson S \& Strang J. Frequency of non-fatal heroin overdose: survery of heroin users recruited in non-clinical settings. BMJ. 1996; 313: 402

18. Taylor A, Frischer M \& Goldberg D. Non-fatal overdosing is related to polydrug use in Glasgow. BMJ. 1996; 13: 1400-01

19. Delgado Rodriguez M. Discordancias entre los estudios de ámbitos hospitalario y comunitario cuando evalúan la misma pregunta de investigación. Gac Sanit. 2002; 16: 344-53.

20. Zador D, Sunjic S, Darke S. Heroin-related deaths in New South Wales, 1992: toxicological findings and circumstances. Med J Aust. 1996; 164: 204-7

21 Darke S, Sunjic S, Zador D, Prolov T. A comparison of blood toxicology of heroin-related deaths and current heroin users in Sydney, Australia. Drug Alcohol Depend 1997; 22: 718-24.

22. Steentoft A, Worm K, Pedersen CB, Sprehn M, Mogensen T, Sorensen MB, ET AL. Drugs in blood samples from unconscious drug addicts after the intake of an overdose. Int J Legal Med 1996; 108: 248-51.

23. Gutiérrez-Cebollada J, de la Torre R, Ortuño J, Garcés JM, Camí J. Psycotropic drug consumption and other factors associated with heroin overdose. Drug Alcohol Depend 1994 Apr;35(2): 16974.

24. Drummer O, Staikos V \& Gerostamoulos J. Heroin-related deaths in Victoria, Australia. Turning Point Alcohol and Drug Centre. Victoria: TPADC; 1999.

25. Strang J, Powis B, Best D, et al. Preventing opiate overdose fatalities with take-home naloxone. Addiction. 1999; 94: 199-204.

26. Strang J, Best D, Man L, Noble A, Gossop M. Peerinitiated overdose resucitation: fellow drug users could be mobilised to implement resucitation. Int $\mathrm{J}$ of Drug Policy 2000; 11: 437-45. 